\title{
Dynamics of Early Serum Tumour Markers and Neutrophil-to-Lymphocyte Ratio Predict Response to PD-I/PD-LI Inhibitors in Advanced Non-Small-Cell Lung Cancer
}

\begin{abstract}
Yin Tang, ${ }^{1,2, *}$ Yu Cui, ${ }^{3-5, *}$ Lin-lin Li, 6,* Ya-ping Guan, (iD ${ }^{3-5}$ Dong-feng Feng,, ${ }^{3-5}$ Bei-bei Yin, ${ }^{3-5}$ Xue-feng Liang, ${ }^{2}$ Jing Yin, ${ }^{2}$ Rui Jiang, Jing Liang, (D) ${ }^{3-5}$ Ya-hong Sun, ${ }^{2}$ Jun Wang ${ }^{3-5}$

'Postgraduate Department, Shandong First Medical University \& Shandong Academy of Medical Sciences, Jinan, 250117, People's

Republic of China; ${ }^{2}$ Department of

Oncology, The Third Affiliated Hospital of

Shandong First Medical University (Affiliated

Hospital of Shandong Academy of Medical

Sciences), Jinan, 25003I, People's Republic of China; ${ }^{3}$ Department of Oncology, The First Affiliated Hospital of Shandong First Medical University \& Shandong Provincial Qianfoshan Hospital, Jinan, 2500 I4, People's Republic of China; ${ }^{4}$ Shandong Lung Cancer Institute, Jinan, 2500 I4, People's Republic of China; ${ }^{5}$ Shandong Key Laboratory of Rheumatic Disease and Translational Medicine, Jinan, 2500I4, People's Republic of China; ${ }^{6}$ Department of Oncology, Shandong Provincial Hospital Affiliated to Shandong First Medical University, Jinan, 25002I, People's Republic of China
\end{abstract}

*These authors contributed equally to this work

Correspondence: Ya-hong Sun Department of Oncology, The Third Affiliated Hospital of Shandong First Medical University (Affiliated Hospital of Shandong Academy of Medical Sciences), No. 38, Wu Yingshan Road, Tianqiao District, Jinan, 25003I, People's Republic of China

Tel +86-53I-5862855I

Email sunyahong@sdfmu.edu.cn

Jun Wang

Department of Oncology, The First Affiliated Hospital of Shandong First Medical University \& Shandong Provincial Qianfoshan Hospital, No. 16766, Jingshi Road, Lixia District, Jinan, 2500 I4, People's Republic of China

Tel +86-53I-89268839

Email ggjun2005@I26.com
Purpose: To evaluate the dynamics of early serum tumour markers (STMs) and the neutrophil-to-lymphocyte ratio (NLR) to predict clinical efficacy and prognosis of advanced non-small-cell lung cancer (NSCLC) patients who received programmed cell death-1 (PD-1)/ programmed cell death-ligand 1 (PD-L1) inhibitors.

Patients and Methods: We retrospectively reviewed patients with advanced NSCLC treated with PD-1/PD-L1 inhibitors between September 2017 and August 2020. NLR and STMs were routinely measured between immunotherapy initiation and the first radiological evaluation. A combination score based on the leading STM and NLR and their dynamic changes was established. The effects of leading STM change, NLR change, and the combination score on the objective response rate (ORR), durable clinical benefit (DCB), progression-free survival (PFS), and overall survival (OS) were analysed. The accuracy of the combination score was evaluated by receiver operating characteristic (ROC) curve and the area under the curve (AUC).

Results: Overall, 124 patients were included in this retrospective cohort study. The ORR was $22.8 \%$, DCB was 54.5\%, and the median OS and PFS were 21.6 and 14.9 months, respectively. Patients with low combination scores had a significantly improved ORR and DCB compared with those with intermediate or high scores $(P=0.002$ for ORR, $P<0.0001$ for DCB). In a multivariate model, the combination score was an independent indicator of PFS $(P<0.0001)$ and OS $(P<0.0001)$. The AUC demonstrated that the combination score $(\mathrm{AUC}=0.706)$ has greater predictive power than either the posttreatment NLR (AUC = 0.668 ) or the leading STM change (AUC $=0.648$ ) alone.

Conclusion: An easy, cost-effective, and novel combination score based on the dynamics of an early STM and the NLR can accurately predict the clinical efficacy of PD-1/PD-L1 inhibitors and prognosis in advanced NSCLC patients.

Keywords: serum tumour marker, neutrophil-to-lymphocyte ratio, PD-1 inhibitors, nonsmall-cell lung cancer, prognosis

\section{Introduction}

Lung cancer has the highest mortality rate among all cancers worldwide and in China and accounts for $23 \%$ of all cancer-related deaths. Approximately $75 \%$ to $80 \%$ of all lung cancer cases are non-small-cell lung cancer (NSCLC), which usually presents as advanced or metastatic disease at initial diagnosis. ${ }^{1-3}$ Immunotherapy consisting of immune checkpoint inhibitor (ICI) blockade, and 
specifically programmed cell death-ligand 1 (PD-L1) or programmed cell death-1 (PD-1) inhibitors, has revolutionized therapeutic strategies for advanced human cancers over the last few years. Durable responses and improved survival can be observed in advanced NSCLC patients treated with ICIs as first- or second-line treatments, as can tolerated immune-related adverse events. However, by far, only a minority of patients initially respond to ICI administration. Although the expression status of PDL1 by immunohistochemistry has been widely used to identify pretreatment NSCLC patients who might benefit from such therapies, patients with negative PD-L1 staining may still be responsive. Other biomarkers, such as tumour-infiltrating lymphocytes (TILs), the Teff score, which represents effector T-cell gene expression, tumour mutational burden (TMB, the number of somatic mutations within the coding region of a tumour genome), and tumour neoantigen burden (TNB, the number of tumourspecific antigens generated by somatic mutations that produce novel peptides), have been proposed. ${ }^{4-8}$ However, analysis of these biomarkers is expensive and time-consuming, and to some extent, a consensus regarding detection and analytical methods is lacking, as this approach has not yet been widely applied in clinical practice. Thus, the development of inexpensive and repeatable tests with easily obtainable results for biomarkers is vital to optimize the clinical use of ICIs, to identify a subset of patients who are most likely to derive a clinical benefit, and to help understand the potential molecular determinants of an immune response.

Recent studies have indicated that predictors in the host could help select patients for ICI therapy, including Eastern Cooperative Oncology Group (ECOG) performance status, routine laboratory parameters, such as lactate dehydrogenase and peripheral blood cell counts, and gut microbiota. ${ }^{9-11}$ Inflammation and the inflammatory response have been viewed as surrogate biomarkers of host immunity. Neutrophilia was found to occur in an inflammatory response where it suppresses the cytolytic activity of immune cells, including lymphocytes, natural killer cells, and activated T cells. An elevated neutrophil-to-lymphocyte ratio (NLR) has been reported to be tightly associated with systemic inflammation and the immune status of the host and has been extensively investigated in solid tumours as a poor prognostic factor. ${ }^{12-14}$ Serum tumour markers (STMs), surrogate indicators of tumour burden, such as carcinoembryonic antigen (CEA), carbohydrate antigen
19-9 (CA19-9), and cytokeratin-19 fragments (CYFRA 21-1), are also prognostic indicators in NSCLC patients with or without immune checkpoint blockade treatment. ${ }^{15-}$

${ }^{17}$ Recently, Lang et al reported that STM dynamics could aid in evaluating the therapeutic outcomes of ICI treatment in NSCLC patients, but the enrichment of clinical response seemed to be incomplete. ${ }^{17}$

In this study, a novel scoring system incorporating the dynamics of early STMs and the NLR was developed, and its predictive role in the efficacy of ICI therapy and prognosis in NSCLC patients was analysed. Early changes in the combination of markers provide clinicians with predictive and prognostic information as to which patients with advanced NSCLC may benefit from ICI treatment.

\section{Patients and Methods Patients and Data Collection}

We performed a retrospective analysis of all patients with a histologically or cytologically proven diagnosis of advanced NSCLC who were treated with a PD-1 or PDL1 inhibitor at No. 960 Hospital, People's Liberation of Army, the First Affiliated Hospital of Shandong First Medical University, and the Third Affiliated Hospital of Shandong First Medical University from September 2017 to August 2020. This study was approved by the independent research ethics committee of The First Affiliated Hospital of Shandong First Medical University (NO: YXLL-KY-2020-007) and conformed to the principles of the Declaration of Helsinki.

The inclusion criteria were as follows: age older than 18 years; histologically or cytologically confirmed NSCLC; stage IIIB or IV disease (locally advanced or metastatic) according to the American Joint Committee on Cancer (AJCC) staging system, 8th version; treatment with PD-1 inhibitor (nivolumab, pembrolizumab, camrelizumab, sintilimab, or tislelizumab) or PD-L1 inhibitor (durvalumab or atezolizumab) monotherapy or immunotherapy combined with chemotherapy as a first-, secondor later line of treatment; an Eastern Cooperative Oncology Group (ECOG) performance status of 0-2, and at least 2 treatment cycles. We excluded patients who were obviously in a chronic or acute inflammatory state. All included patients had measurable disease and were treated until disease progression or until unacceptable toxicity was reached. Efficacy evaluation by radiographic findings was performed according to Response Evaluation Criteria in Solid Tumours version 1.1 (RECIST 1.1) and included 
complete response (CR), partial response (PR), objective response rate (ORR), and durable clinical benefit (DCB).

The clinical characteristics of the patients were collected from the electronic medical records and included age, sex, body mass index (BMI), histology, and tumour node metastasis (TNM) stage. Peripheral blood data, including absolute neutrophil count, absolute lymphocyte count and the levels of CEA, CYFRA 21-1, CA19-9, neuron-specific enolase (NSE) and carbohydrate antigen 125 (CA125), were predominantly used to compute the NLR and leading STM at the start of treatment and at 6 weeks after treatment initiation. The upper limits of normal were $5 \mathrm{ng} / \mathrm{mL}$ for CEA, $3.3 \mathrm{ng} / \mathrm{mL}$ for CYFRA 21-1, $16.3 \mathrm{ng} / \mathrm{mL}$ for NSE, $37 \mathrm{U} / \mathrm{mL}$ for CA19-9, and $35 \mathrm{U} / \mathrm{L}$ for CA125. If more than one STM increased upon ICI initiation, the STM with the highest elevation relative to the upper limit of normal, according to previously reported criteria, was selected as the leading STM. ${ }^{17}$ If no analysed STMs were elevated at immunotherapy initiation, the leading STM was the one with the highest value in relation to the upper limit of normal. The NLR was calculated by dividing the absolute neutrophil counts by the lymphocyte counts, as measured in peripheral blood.

\section{Statistical Analysis}

Categorical variables, such as patient and disease characteristics, were described using frequencies and percentages. Quantitative variables are presented as medians and ranges. Durable clinical benefit (DCB) was defined as ICI continuation for six months. Durable clinical benefit (DCB) was defined as CR, PR or stable disease (SD) that lasted longer than 6 months. Progression-free survival (PFS) was defined as the time from immunotherapy initiation to the date of disease progression or death from any cause, whichever occurred first. Patients who were alive without disease progression were censored on the date of their last disease assessment. Overall survival (OS) was defined as the time from immunotherapy initiation to death from any cause. Patients who were still alive were censored at the date of last contact. PFS and OS curves were calculated using the Kaplan-Meier method and were compared using the Log rank test. Cox regression models were applied to determine independent indicators associated with PFS and OS and to estimate the hazard ratio (HR) and $95 \%$ confidence interval (CI). Factors that were statistically significant in the univariate analysis were incorporated into the multivariate analysis. SPSS 26.0 software (SPSS Inc., Chicago, IL) was used for all statistical tests.
A $P$ value $<0.05$ was considered statistically significant. The accuracy of the combination score was evaluated by an ROC curve and by calculating the AUC.

\section{Results}

\section{Clinicopathological Characteristics of the Patients}

We initially reviewed the data of 141 advanced NSCLC patients treated with immunotherapy, and finally, 124 patients were included in this analysis. 17 patients were excluded because they were lost to follow-up $(n=11)$ or received fewer than 2 cycles of therapy $(n=6)$. The median age was 63 years (range 36 to 85 years), and $28.2 \%$ of patients were female. 37 patients received ICI monotherapy, while 87 patients were treated with chemotherapy plus immunotherapy. In the total population, CEA was determined to be the leading STM in 57 (51.4\%), CYFRA 211 in $36(32.4 \%)$, NSE in 12 (10.8\%), CA125 in 4 (3.6\%), and CA19-9 in $2(1.8 \%)$ patients. In 13 cases, no STM was elevated at the time of ICI initiation. Dynamics of the leading STM were assessed in 111 (89.5\%) patients. The baseline clinicopathological and laboratory characteristics are summarized in Table 1. At the end of follow-up, 80 patients had relapsed and 38 patients had died. The median follow-up duration was 10.8 months (95\% CI: 7.5-14.1 months). For the entire population, the ORR was $22.8 \%$, DCB was 54.5\%, and the median OS and PFS were 21.6 months and 14.9 months, respectively.

\section{Association Between Leading STM or NLR Dynamics and Response to ICl Treatment and Outcomes}

We first analysed the association between the dynamics of the leading STM or those of the NLR and response to ICI treatment and patient outcomes. A decreased leading STM was defined as a leading STM that decreased more than $20 \%$ compared with the baseline leading STM at 6 weeks after ICI initiation, while an increased leading STM was defined as a leading STM that had not decreased more than $20 \%$ over the same period. No clinicopathological or laboratory characteristics were associated with leading STM dynamics except for ECOG performance status $(P=0.05)$ (Table 2$)$. As shown in Figure 1 , the ORR was significantly higher in the decreased leading STM group than in the increased leading STM group $(32.0 \%$ vs $8.0 \%, P=0.003)$. Similarly, the difference in DCB between the two groups was significant $(64.0 \%$ vs 
Table I Baseline Characteristics of Patients

\begin{tabular}{|c|c|c|}
\hline Median Age Years (Range) & & $63(36-85)$ \\
\hline \multirow[t]{2}{*}{ Age (n, \%) } & $<65$ years & $73(58.87)$ \\
\hline & $\geq 65$ years & $5 I(4 I .13)$ \\
\hline \multirow[t]{2}{*}{ Gender (n, \%) } & Female & $35(28.23)$ \\
\hline & Male & 89 (7I.77) \\
\hline \multirow[t]{3}{*}{ BMI (n, \%) } & $<18.5 \mathrm{~kg} / \mathrm{m}^{2}$ & $3(2.46)$ \\
\hline & $18.5-23.9 \mathrm{~kg} / \mathrm{m}^{2}$ & $61(50.00)$ \\
\hline & $>23.9 \mathrm{~kg} / \mathrm{m}^{2}$ & $58(47.54)$ \\
\hline \multirow[t]{5}{*}{ Histology (n, \%) } & Adenocarcinoma & $70(56.45)$ \\
\hline & Squamous carcinoma & $40(32.26)$ \\
\hline & Adenosquamous carcinoma & $3(2.42)$ \\
\hline & Large cell carcinoma & $5(4.03)$ \\
\hline & Unknown & $6(4.84)$ \\
\hline \multirow[t]{2}{*}{ TNM stage (n, \%) } & III & $32(25.8 I)$ \\
\hline & IV & $92(74.19)$ \\
\hline \multirow[t]{3}{*}{ ECOG PS (n, \%) } & 0 & $25(20.16)$ \\
\hline & I & $98(79.03)$ \\
\hline & 2 & $\mathrm{I}(0.8 \mathrm{I})$ \\
\hline \multirow[t]{3}{*}{ Smoking status (n, \%) } & Never & $46(37.10)$ \\
\hline & Former & $59(47.58)$ \\
\hline & Current & $19(15.32)$ \\
\hline \multirow[t]{6}{*}{ Metastasis sites (n, \%) } & Bone & 14 (I4.89) \\
\hline & Lymph node & $30(31.91)$ \\
\hline & Lung & $36(38.30)$ \\
\hline & Brain & $7(7.45)$ \\
\hline & Liver & $4(4.26)$ \\
\hline & Adrenal gland & $3(3.19)$ \\
\hline \multirow[t]{2}{*}{ Radiotherapy (n, \%) } & Yes & $44(35.48)$ \\
\hline & No & $80(64.52)$ \\
\hline \multirow[t]{4}{*}{ PD-LI expression (n, \%) } & Not available & 89 (7I.77) \\
\hline & $<50 \%$ & $21(16.94)$ \\
\hline & $\mathrm{I}-49 \%$ & $10(8.06)$ \\
\hline & Negative & $4(3.23)$ \\
\hline \multirow[t]{3}{*}{ Line of immunotherapy (n, \%) } & I & $31(25.00)$ \\
\hline & 2 & $63(50.81)$ \\
\hline & $\geq 3$ & $30(24.19)$ \\
\hline \multirow[t]{2}{*}{$\mathrm{ICl}(\mathrm{n}, \%)$} & PD-I & $107(86.29)$ \\
\hline & PD-LI & $17(13.7 \mid)$ \\
\hline \multirow[t]{2}{*}{ Treatment type (n, \%) } & Monotherapy & $37(29.84)$ \\
\hline & Combination therapy & $87(70.16)$ \\
\hline \multirow[t]{2}{*}{ Immune-related adverse events (n, \%) } & Yes & 119 (95.97) \\
\hline & No & $5(4.03)$ \\
\hline \multirow[t]{2}{*}{ Leading STM (n, \%) } & CEA & $57(51.35)$ \\
\hline & CYFRA 2I-I & $36(32.43)$ \\
\hline
\end{tabular}

(Continued) 
Table I (Continued).

\begin{tabular}{|c|c|c|}
\hline & $\begin{array}{l}\text { NSE } \\
\text { CAI9-9 } \\
\text { CAI25 }\end{array}$ & $\begin{array}{l}12(10.8 I) \\
2(1.80) \\
4(3.6 I)\end{array}$ \\
\hline Baseline CEA (n, \%) & $\begin{array}{l}\text { Normal (<5 ng/mL) } \\
\text { Increased ( } \geq 5 \mathrm{ng} / \mathrm{mL})\end{array}$ & $\begin{array}{l}47(43.52) \\
61(56.48)\end{array}$ \\
\hline Baseline CYFRA 2I-I (n, \%) & $\begin{array}{l}\text { Normal }(<3.3 \mathrm{ng} / \mathrm{mL}) \\
\text { Increased }(\geq 3.3 \mathrm{ng} / \mathrm{mL})\end{array}$ & $\begin{array}{l}23(24.47) \\
71(75.53)\end{array}$ \\
\hline Baseline NSE (n, \%) & $\begin{array}{l}\text { Normal }(<16.3 \mathrm{ng} / \mathrm{mL}) \\
\text { Increased }(\geq 16.3 \mathrm{ng} / \mathrm{mL})\end{array}$ & $\begin{array}{l}49(56.98) \\
37(43.02)\end{array}$ \\
\hline Baseline CAI9-9 (n, \%) & $\begin{array}{l}\text { Normal }(<37 \mathrm{U} / \mathrm{mL}) \\
\text { Increased }(\geq 37 \mathrm{U} / \mathrm{mL})\end{array}$ & $\begin{array}{l}13(59.09) \\
9(40.91)\end{array}$ \\
\hline Baseline CAI25 (n, \%) & $\begin{array}{l}\text { Normal (<35 U/L) } \\
\text { Increased ( } \geq 35 \mathrm{U} / \mathrm{L})\end{array}$ & $\begin{array}{l}20(54.05) \\
17(45.95)\end{array}$ \\
\hline Baseline NLR (n, \%) & $\begin{array}{l}\text { Decreased }(<5) \\
\text { Increased }(\geq 5)\end{array}$ & $\begin{array}{l}97(78.23) \\
27(21.77)\end{array}$ \\
\hline
\end{tabular}

Note: Data are given as absolute number and percent within the respective group unless otherwise specified.

Abbreviations: BMI, body mass index; TNM, tumor node metastasis; ECOG PS, Eastern Cooperative Oncology Group performance status; ICl, immune checkpoint inhibitor; PD-I, programmed cell death-I; PD-LI, programmed cell death-ligand I; STM, serum tumor markers; CEA, carcinoembryonic antigen; CYFRA 2I-I, cytokeratin19 fragments; NSE, neuron specific enolase; CAI9-9, carbohydrate antigen 19-9; CAI25, carbohydrate antigen I25.

$26.0 \%, P=0.0001)$. The patients with increased leading STM had a lower median PFS (9.5 vs 19.1 months, HR: 4.123, 95\% CI: $1.832-9.430, P=0.0002)$ and OS (13.9 vs not reached months, HR: $3.526,95 \% \mathrm{CI}: 1.570-7.921, P=$ 0.0015 ) than those with decreased leading STM (Figure 2). In terms of NLR dynamics, a decreased NLR was defined as a NLR that had decreased more than $20 \% 6$ weeks after ICI initiation compared with the baseline NLR, while an increased NLR was defined as a NLR that had not decreased more than $20 \% 6$ weeks after ICI initiation compared with the baseline. No significant difference was observed between the decreased NLR group and the increased NLR group in DCB, PFS and OS (Supplementary Figure 1). Thus, the decreased NLR and increased NLR were redefined as posttreatment NLR $<5$ and NLR $\geq 5$, respectively, regardless of the pretreatment NLR. As expected, patients with decreased NLR were more likely to benefit from ICI treatment (for ORR, $27.3 \%$ vs $4.2 \%, P=0.015$; for $\mathrm{DCB}, 52.5 \%$ vs $16.7 \%$, $P=0.002$ ) (Figure 1). Furthermore, patients with an increased NLR were more likely to experience progression (19.1 vs 3.1 months, HR: 6.161, 95\% CI: 3.215-11.810, $P<0.0001)$ or die (21.7 vs 5.0 months, HR: 4.340, 95\% CI: 2.265-8.317, $P<0.0001$ ) (Figure 2).

\section{Association Between a Combination Score of the Leading STM and NLR Dynamics and Response to ICl Treatment and Outcomes}

We next asked whether a combination score incorporating the leading STM and NLR dynamics could accurately predict response to ICI treatment and patient outcomes. A combination score (score 0: low combination score; score 1: intermediate; score 2: high combination score) was established by combining the dynamics of the leading STM (score 0: decreased leading STM; score 1: increased leading STM) and the NLR (score 0: posttreatment NLR < 5; score 1: posttreatment NLR $\geq 5$ ).

With the total population as the overall cohort $(n=100)$, patients with a low combination score had a significantly improved ORR (34.8\% vs $10.3 \%$ vs $0 \%, P=0.002)$ and DCB $(69.6 \%$ vs $28.2 \%$ vs $13.3 \%, P<0.0001)$ compared with those with intermediate or high combination scores (Figure 1). In the cohort of the Third Affiliated Hospital of Shandong First Medical University $(n=40)$, however, no significant difference in the ORR or DCB was observed among these three groups (Figure 1). In the subgroup of nonresponders (SD and progressive disease), patients with 
Table 2 Associations Between Clinicopathological Features and Leading STM, NLR, and Combination Score

\begin{tabular}{|c|c|c|c|c|c|c|c|c|c|c|c|}
\hline \multirow[t]{2}{*}{ Variables } & \multirow[t]{2}{*}{ Category } & \multicolumn{2}{|c|}{ Leading STM } & \multirow[t]{2}{*}{$\mathbf{P}$} & \multicolumn{2}{|l|}{ NLR } & \multirow[t]{2}{*}{$\mathbf{P}$} & \multicolumn{3}{|c|}{$\begin{array}{l}\text { Combination } \\
\text { Score }\end{array}$} & \multirow[t]{2}{*}{$\mathbf{P}$} \\
\hline & & Decreased & Increased & & Decreased & Increased & & 0 & $\mathbf{I}$ & 2 & \\
\hline Age (years) & $\begin{array}{l}<65 \\
\geq 65\end{array}$ & $\begin{array}{l}24 \\
26\end{array}$ & $\begin{array}{l}33 \\
17\end{array}$ & 0.069 & $\begin{array}{l}58 \\
42\end{array}$ & $\begin{array}{l}15 \\
9\end{array}$ & 0.687 & $\begin{array}{l}21 \\
25\end{array}$ & $\begin{array}{l}28 \\
11\end{array}$ & $\begin{array}{l}8 \\
7\end{array}$ & 0.050 \\
\hline Gender & $\begin{array}{l}\text { Female } \\
\text { Male }\end{array}$ & $\begin{array}{l}11 \\
39\end{array}$ & $\begin{array}{l}16 \\
34\end{array}$ & 0.260 & $\begin{array}{l}29 \\
71\end{array}$ & $\begin{array}{l}6 \\
18\end{array}$ & 0.696 & $\begin{array}{l}11 \\
35\end{array}$ & $\begin{array}{l}12 \\
27\end{array}$ & $\begin{array}{r}4 \\
11\end{array}$ & 0.828 \\
\hline BMI $\left(\mathrm{kg} / \mathrm{m}^{2}\right)$ & $\begin{array}{l}18.5-23.9 \\
<18.5 \text { or }>23.9\end{array}$ & $\begin{array}{l}26 \\
24\end{array}$ & $\begin{array}{l}21 \\
27\end{array}$ & 0.414 & $\begin{array}{l}47 \\
52\end{array}$ & $\begin{array}{l}14 \\
9\end{array}$ & 0.247 & $\begin{array}{l}24 \\
22\end{array}$ & $\begin{array}{l}15 \\
23\end{array}$ & $\begin{array}{l}8 \\
6\end{array}$ & 0.387 \\
\hline Histology & $\begin{array}{l}\text { Adenocarcinoma } \\
\text { Non- } \\
\text { adenocarcinoma }\end{array}$ & $\begin{array}{l}31 \\
15\end{array}$ & $\begin{array}{l}27 \\
22\end{array}$ & 0.220 & $\begin{array}{l}62 \\
32\end{array}$ & $\begin{array}{l}8 \\
16\end{array}$ & 0.004 & $\begin{array}{l}30 \\
12\end{array}$ & $\begin{array}{l}22 \\
16\end{array}$ & $\begin{array}{l}6 \\
9\end{array}$ & 0.088 \\
\hline TNM stage & $\begin{array}{l}\text { III } \\
\text { IV }\end{array}$ & $\begin{array}{l}14 \\
36\end{array}$ & $\begin{array}{l}13 \\
37\end{array}$ & 0.822 & $\begin{array}{l}29 \\
71\end{array}$ & $\begin{array}{l}3 \\
21\end{array}$ & 0.097 & $\begin{array}{l}13 \\
33\end{array}$ & $\begin{array}{l}13 \\
26\end{array}$ & $\begin{array}{r}1 \\
14\end{array}$ & 0.143 \\
\hline ECOG PS & $\begin{array}{l}0 \\
\geq 1\end{array}$ & $\begin{array}{l}11 \\
39\end{array}$ & $\begin{array}{l}4 \\
46\end{array}$ & 0.050 & $\begin{array}{l}21 \\
79\end{array}$ & $\begin{array}{l}4 \\
20\end{array}$ & 0.781 & $\begin{array}{l}11 \\
35\end{array}$ & $\begin{array}{r}2 \\
37\end{array}$ & $\begin{array}{r}2 \\
13\end{array}$ & 0.040 \\
\hline Smoking status & $\begin{array}{l}\text { Never } \\
\text { Former/Current }\end{array}$ & $\begin{array}{l}17 \\
33\end{array}$ & $\begin{array}{l}20 \\
30\end{array}$ & 0.534 & $\begin{array}{l}37 \\
63\end{array}$ & $\begin{array}{l}9 \\
15\end{array}$ & 0.964 & $\begin{array}{l}16 \\
30\end{array}$ & $\begin{array}{l}14 \\
25\end{array}$ & $\begin{array}{l}7 \\
8\end{array}$ & 0.698 \\
\hline Metastasis & $\begin{array}{l}\text { No } \\
\text { Yes }\end{array}$ & $\begin{array}{l}12 \\
38\end{array}$ & $\begin{array}{l}13 \\
37\end{array}$ & 0.817 & $\begin{array}{l}27 \\
73\end{array}$ & $\begin{array}{l}3 \\
21\end{array}$ & 0.136 & $\begin{array}{l}11 \\
35\end{array}$ & $\begin{array}{l}13 \\
26\end{array}$ & $\begin{array}{r}1 \\
14\end{array}$ & 0.130 \\
\hline Radiotherapy & $\begin{array}{l}\text { Yes } \\
\text { No }\end{array}$ & $\begin{array}{l}17 \\
33\end{array}$ & $\begin{array}{l}18 \\
32\end{array}$ & 0.834 & $\begin{array}{l}31 \\
69\end{array}$ & $\begin{array}{l}13 \\
11\end{array}$ & 0.033 & $\begin{array}{l}16 \\
30\end{array}$ & $\begin{array}{r}9 \\
30\end{array}$ & $\begin{array}{r}10 \\
5\end{array}$ & 0.011 \\
\hline PD-LI expression & $\begin{array}{l}<50 \% \\
\geq 50 \%\end{array}$ & $\begin{array}{l}7 \\
8\end{array}$ & $\begin{array}{l}7 \\
2\end{array}$ & 0.210 & $\begin{array}{l}18 \\
10\end{array}$ & $\begin{array}{l}3 \\
0\end{array}$ & 0.533 & $\begin{array}{l}7 \\
8\end{array}$ & $\begin{array}{l}6 \\
2\end{array}$ & $\begin{array}{l}1 \\
0\end{array}$ & 0.388 \\
\hline PD-LI status & $\begin{array}{l}\text { Positive } \\
\text { Negative }\end{array}$ & $\begin{array}{l}15 \\
3\end{array}$ & $\begin{array}{l}9 \\
1\end{array}$ & 1.000 & $\begin{array}{l}27 \\
4\end{array}$ & $\begin{array}{l}5 \\
0\end{array}$ & 1.000 & $\begin{array}{r}14 \\
3\end{array}$ & $\begin{array}{l}8 \\
1\end{array}$ & $\begin{array}{l}2 \\
0\end{array}$ & 1.000 \\
\hline Line of immunotherapy & $\begin{array}{l}<3 \\
\geq 3\end{array}$ & $\begin{array}{l}37 \\
13\end{array}$ & $\begin{array}{l}36 \\
14\end{array}$ & 0.822 & $\begin{array}{l}75 \\
25\end{array}$ & $\begin{array}{l}19 \\
5\end{array}$ & 0.669 & $\begin{array}{l}35 \\
11\end{array}$ & $\begin{array}{l}26 \\
13\end{array}$ & $\begin{array}{r}12 \\
3\end{array}$ & 0.588 \\
\hline $\mathrm{ICI}$ & $\begin{array}{l}\text { PD-I } \\
\text { PD-LI }\end{array}$ & $\begin{array}{l}45 \\
5\end{array}$ & $\begin{array}{l}44 \\
6\end{array}$ & 0.749 & $\begin{array}{l}88 \\
12\end{array}$ & $\begin{array}{l}19 \\
5\end{array}$ & 0.320 & $\begin{array}{r}41 \\
5\end{array}$ & $\begin{array}{r}36 \\
3\end{array}$ & $\begin{array}{r}12 \\
3\end{array}$ & 0.467 \\
\hline Mono-immunotherapy & $\begin{array}{l}\text { Yes } \\
\text { No }\end{array}$ & $\begin{array}{l}13 \\
37\end{array}$ & $\begin{array}{l}10 \\
40\end{array}$ & 0.476 & $\begin{array}{l}28 \\
72\end{array}$ & $\begin{array}{l}9 \\
15\end{array}$ & 0.361 & $\begin{array}{l}13 \\
33\end{array}$ & $\begin{array}{r}5 \\
34\end{array}$ & $\begin{array}{r}5 \\
10\end{array}$ & 0.124 \\
\hline Immune-related adverse events & $\begin{array}{l}\text { Yes } \\
\text { No }\end{array}$ & $\begin{array}{l}46 \\
4\end{array}$ & $\begin{array}{l}49 \\
1\end{array}$ & 0.362 & $\begin{array}{l}96 \\
4\end{array}$ & $\begin{array}{l}23 \\
1\end{array}$ & 1.000 & $\begin{array}{r}42 \\
4\end{array}$ & $\begin{array}{r}39 \\
0\end{array}$ & $\begin{array}{r}14 \\
1\end{array}$ & 0.132 \\
\hline
\end{tabular}

Notes: Data are given as absolute number and testing for significance between the subgroups was accomplished using a chi square test, a $P$ value of $<0.05$ was regarded statistically significant.

Abbreviations: BMI, body mass index; TNM, tumor node metastasis; ECOG PS, Eastern Cooperative Oncology Group performance status; ICl, immune-checkpoint inhibitors; PD-I, programmed cell death-I; PD-LI, programmed cell death-ligand I; STM, serum tumor markers; NLR, neutrophil-to-lymphocyte ratio.

a low combination score had a significantly improved DCB (66.7\% vs $20.0 \%$ vs $13.3 \%, P<0.0001)$ (Figure 3 ). Moreover, the combination score was significantly associated with PFS (19.1 vs 14.9 vs 2.8 months, $P<0.0001)$ and OS (not reached vs not reached vs 3.1 months, $P<$ 0.0001) in all patients (Figure 2 ) as well as in nonresponders (for PFS, 19.1 vs 9.5 vs 2.8 months, $P<0.0001$; for OS, not reached vs not reached vs 3.1 months, $P<0.0001$ ) (Figure 3 ). This combination score was also further confirmed to stratify patients with different prognoses in terms of PFS (not reached vs not reached vs 2.3 months, $P<0.0001$ ) and OS (not reached vs not reached vs 5 months, $P=0.0003$ ) in the cohort of the Third Affiliated Hospital of Shandong First Medical University $(n=40)$ (Figure 2). 

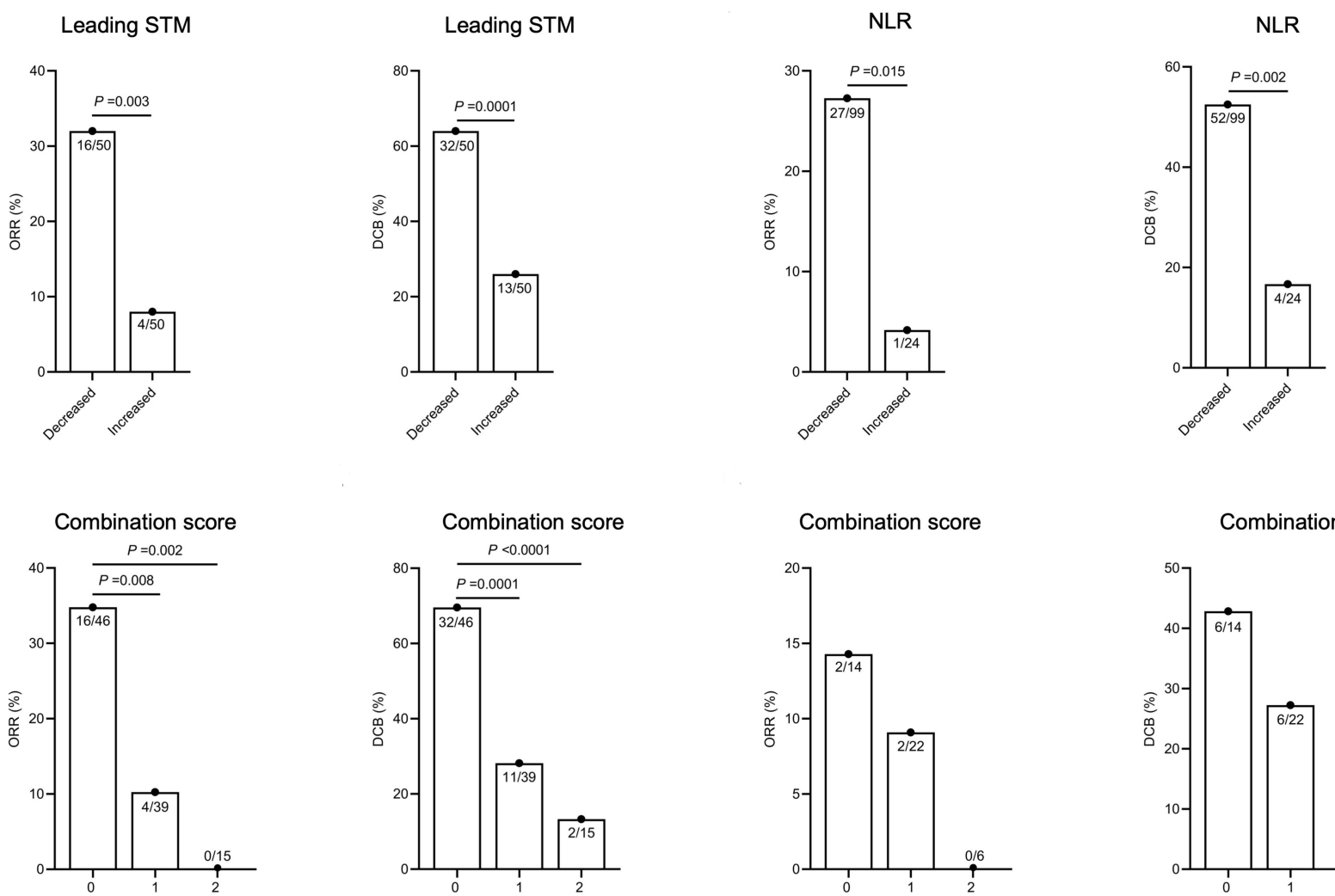

Combination score

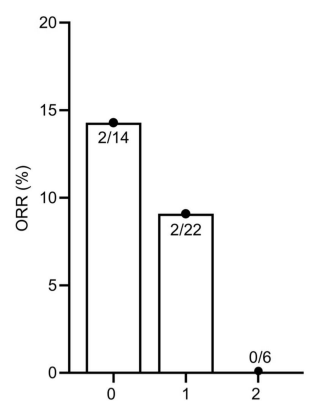

Combination score

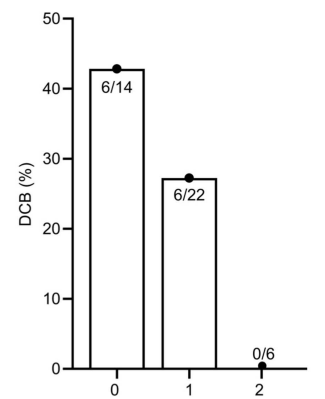

Figure I ORR and DCB from the decreased leading STM versus the increased leading STM, and from the decreased NLR versus the increased NLR. ORR and DCB for patients in the overall cohort with different combination scores, and for patients in the cohort of the Third Affiliated Hospital of Shandong First Medical University with different combination scores.

Abbreviations: STM, serum tumour marker; NLR, neutrophil-to-lymphocyte ratio; ORR, objective response rate; DCB, durable clinical benefit.

In the adenocarcinoma subgroup, significantly improved DCB, PFS and OS were shown in patients with low combination scores (for DCB, $66.7 \%$ vs $31.8 \%$ vs $0 \%, P=0.002$; for PFS, not reached vs 14.9 vs 2.8 months, $P<0.0001$; for OS, not reached vs 16.7 vs 3.1 months, $P<0.0001$ ) (Supplementary Figure 2A and B). In the squamous cell carcinoma subgroup, differences in the ORR among patients with low, intermediate, and high combination scores were significant $(50.0 \%$ vs $0 \%$ vs $0 \%, P=0.002$ ). Additionally, longer median PFS and OS were found in this subgroup of patients with a low combination score (for PFS, 19.1 vs not estimable vs 2.8 months, $P=0.0002$; for OS, 21.7 vs 16.7 vs 5.0 months, $P=0.0001)$ (Supplementary Figure 3A and B). In other subgroups of patients treated with monoimmunotherapy or combination immunotherapy and in those with good or poor ECOG performance status, the predictive potency of the combination score for ORR, DCB, PFS, and OS was also significant (Supplementary Figures 4-7). Figure 4 shows computed tomography scans at baseline and the first and subsequent radiological assessments for 3 representative NSCLC patients treated with PD-1/PD-L1 inhibitors. These patients with different combination scores exhibited different responses and outcomes following ICI treatment.

According to Supplementary Table 1, ECOG PS, leading STM, posttreatment NLR, an NLR that increased $\geq 20 \%$ and the combination score were stronger predictors of these two outcomes (ORR, DCB). The results of the univariate and stepwise multivariate Cox regression analyses for variables influencing PFS and OS are summarized in Tables 3 and 4. Before developing a combination score, the NLR and STM were first incorporated in the multivariable Cox regression model (Table 4A). The posttreatment NLR was an independent indicator of improved OS (HR: 0.122, 95\% CI: 0.042-0.352, $P=0.001$ ) and PFS (HR: $0.144,95 \%$ CI: $0.048-0.427, P=$ 0.001 ), which is shown in Tables 3 and 4A. As shown in Table 4B, the combination score was incorporated in the multivariable Cox regression model and was demonstrated to be an independent predictor. Multivariate analyses revealed 


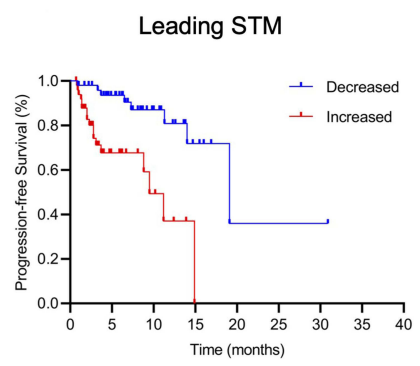

mPFS: 9.5 vs. 19.1 months

HR: $4.123,95 \%$ Cl:1.802-9.430

$P=0.0002$

Combination score

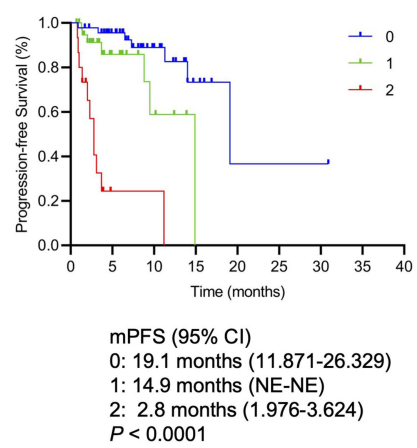

Leading STM

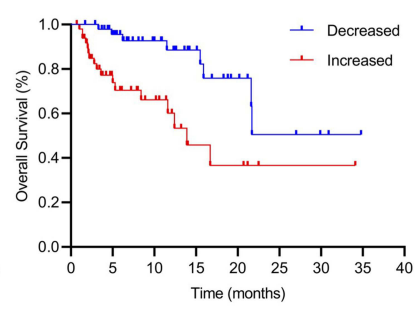

mOS: 13.9 vs. N.R. months HR: $3.526,95 \% \mathrm{Cl}: 1.570-7.921$ $P=0.0015$

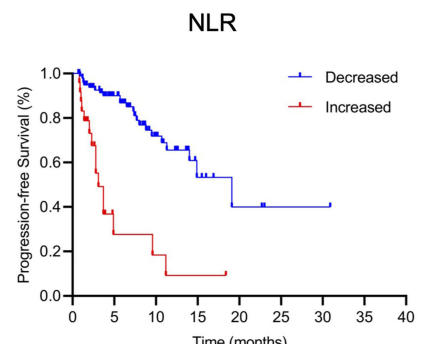

Time (months)

mPFS: 3.1 vs. 19.1 months

HR: $6.161,95 \%$ Cl: 3.215-11.810 $P<0.0001$

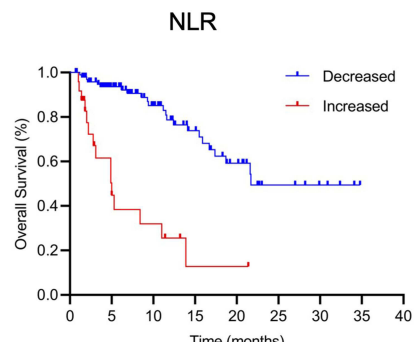

Time (months)

mOS: 5.0 vs. 21.7 months HR: $4.340,95 \%$ Cl: 2.265-8.317 $P<0.0001$
Combination score

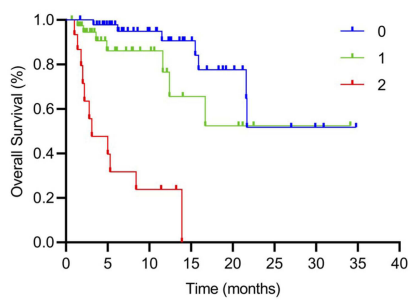

mOS $(95 \% \mathrm{Cl})$

$0:$ NR (NE-NE)

1: NR (NE-NE)

2: 3.1 months $(0.000-6.860)$

$P<0.0001$
Combination score

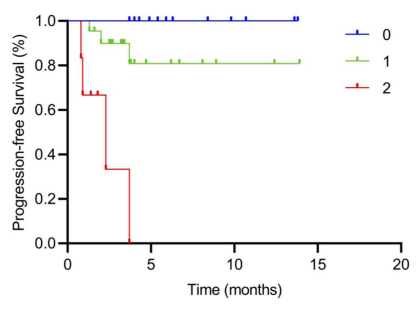

mPFS $(95 \% \mathrm{Cl})$

$0:$ NR (NE-NE)

1: NR (NE-NE)

2: 2.3 months $(0.204-4.396)$

$P<0.0001$
Combination score

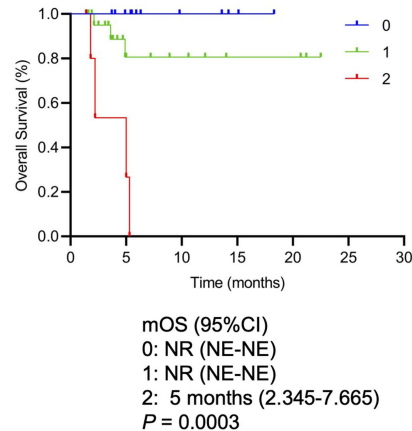

Figure 2 Kaplan-Meier survival curves for progression-free and overall survival according to the leading STM and the NLR. Kaplan-Meier survival curves for progressionfree and overall survival according to the combination scores in the overall cohort and in the cohort of the Third Affiliated Hospital of Shandong First Medical University. Abbreviations: NR, not reached; STM, serum tumour markers; NLR, neutrophil-to-lymphocyte ratio; PFS, progression-free survival; OS, overall survival; HR, hazard ratio; $\mathrm{Cl}$, confidence interval.

\section{A}

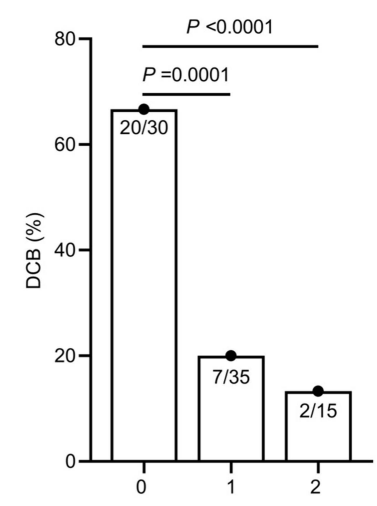

B

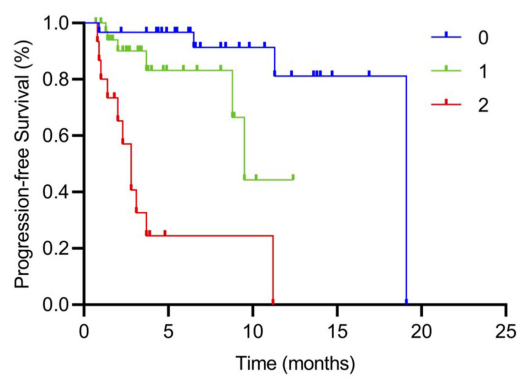

mPFS $(95 \% \mathrm{Cl})$

$0: 19.1$ months (NE-NE)

1: 9.5 months (8.193-20.807)

2: 2.8 months (1.976-3.624)

$P<0.0001$
C

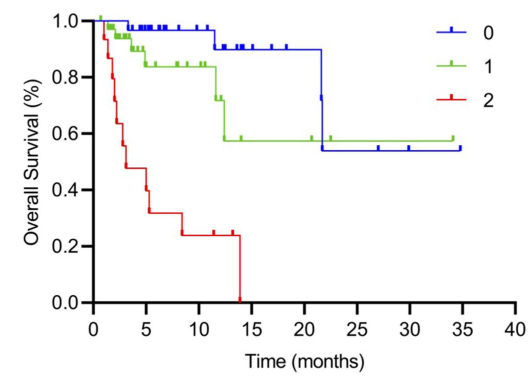

$\operatorname{mOS}(95 \% \mathrm{Cl})$

$0:$ NR (21.7-NE)

1: NR (12.4-NE)

2: 3.1 months $(0-6.860)$

$P<0.0001$

Figure 3 DCB for stable or progressive patients with different combination scores (A). Kaplan-Meier survival curves for progression-free (B) and overall survival (C) according to the combination scores of stable or progressive patients.

Abbreviations: NR, not reached; DCB, durable clinical benefit; PFS, progression-free survival; OS, overall survival; Cl, confidence interval.

a significant effect of the combination score (for the medium score group, HR: $3.661,95 \% \mathrm{CI}: 1.136-11.792, P=0.030$; for the high score group, HR: $20.066,95 \%$ CI: 5.646-71.311, $P<$ $0.0001)$ on PFS. In a multivariate model, the combination score was an independent indicator for predicting OS (for the medium score group, HR: $3.183,95 \%$ CI: $1.02-9.931, P=$ 0.046; for the high score group, HR: $22.299,95 \%$ CI: $6.381-$ 77.924, $P<0.0001)$. To evaluate whether the combination 

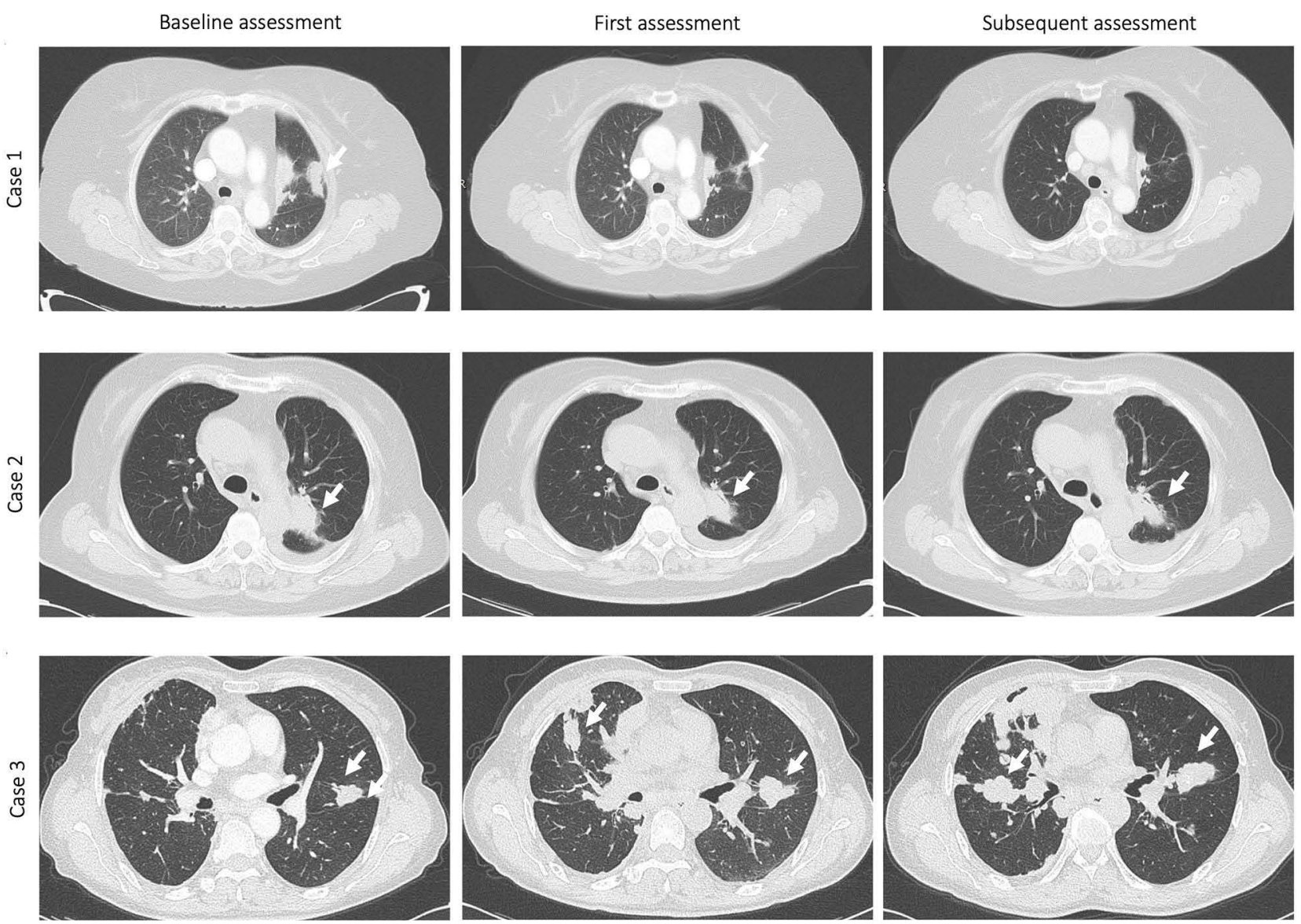

Figure 4 Computed tomography (CT) scans at baseline and at the first and subsequent radiological assessments for 3 representative NSCLC patients treated with PD-I/ PD-LI inhibitors. The first patient (Case I) with a combination score of 0 (low combination score) had achieved a partial response at the first radiological evaluation and subsequently achieved a complete response (at 14 weeks) with long-term survival (alive; progression-free survival: 30.9 months; overall survival: 30.9 months). The second patient (Case 2) with a combination score of 0 (low combination score) had stable disease at the first radiological evaluation and subsequently exhibited a partial response (at 13 weeks) with long-term survival (alive; progression-free survival: 19.1 months; overall survival: 21.7 months). The third patient (Case 3 ) with a combination score of 2 (high combination score) had progressive disease at the first radiological evaluation and subsequently exhibited continuous progression (at 12 weeks) with short-term survival (death; progression-free survival: 2.3 months; overall survival: 5.0 months). The arrows indicate lesions in the lungs.

score is a better predictor than the posttreatment NLR or the leading STM alone, ROC curves were generated, as shown in Figure 5. The AUC value demonstrated that the combination score $(\mathrm{AUC}=0.706)$ as an individual biomarker had greater predictive power than the posttreatment NLR $(\mathrm{AUC}=0.668)$ or the leading STM change (AUC $=0.648$ ).

\section{Discussion}

The present study indicated that combined analysis of the early dynamics of the leading STM and the NLR predicted the efficacy of ICI therapy and outcomes in advanced NSCLC patients. The predictive potency of these combined serum markers showed consistent results in patients with no response at the first radiological assessment. This predictor may also help distinguish patients who might be responsive to immunotherapy from those who are resistant to immunotherapy regardless of their pathologic subtype, ECOG status, or whether they are treated with first-line or late-line ICIs. The AUC value demonstrated that the combination score had greater predictive power than the posttreatment NLR or leading STM change alone.

The high mortality of NSCLC is due to the presence of metastatic disease at the time of diagnosis in most patients, which indicates that improvements in long-term survival will require systemic therapies that are more effective than traditional chemotherapy. ${ }^{18}$ As several PD-1 and PD-L1 inhibitors have been approved as novel therapeutic strategies for the treatment of advanced NSCLC in China and worldwide, ${ }^{19-23}$ immunotherapy alone or in combination with chemotherapy is now the preferred option for patients with metastatic NSCLC. In recent years, however, only $20-30 \%$ of advanced NSCLC patients seem to derive a clinical benefit, and biomarkers that can predict the response to immunotherapy with ICIs remain to be 


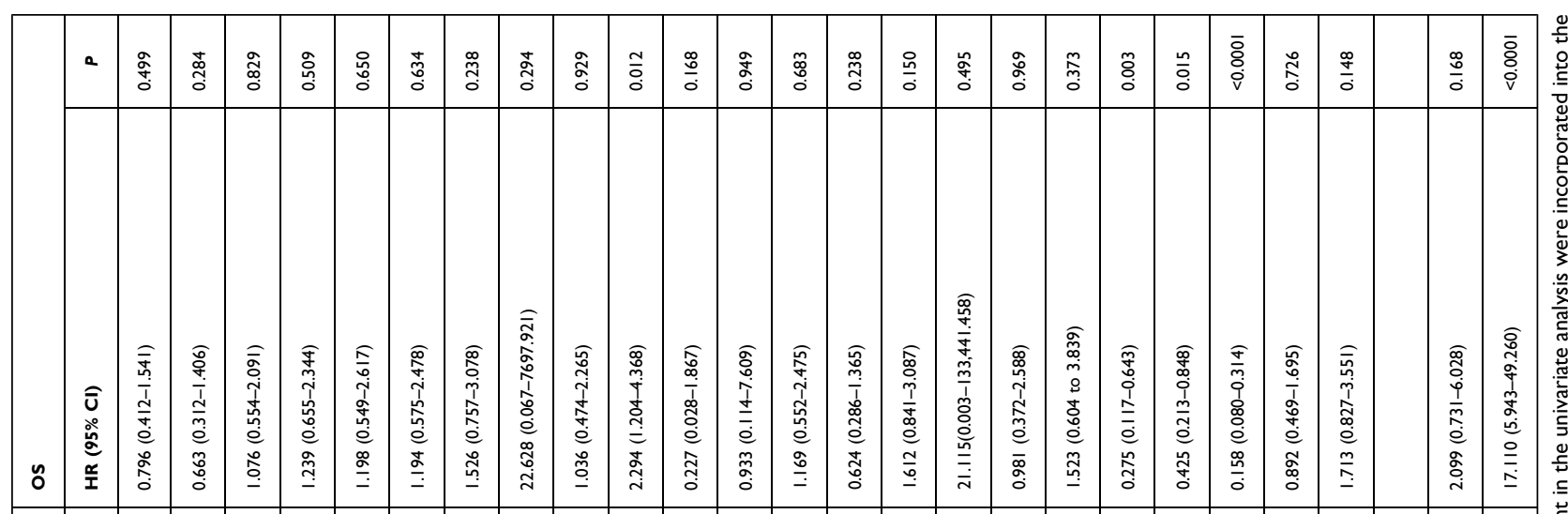


Table 4 Multivariate Cox Analyses of PFS and OS

\begin{tabular}{|c|c|c|c|c|c|}
\hline \multicolumn{2}{|c|}{ A. NLR and STM Multivariate Cox Analysis } & \multicolumn{2}{|l|}{ PFS } & \multicolumn{2}{|l|}{ os } \\
\hline & & HR (95\% Cl) & $\boldsymbol{P}$ & HR (95\% Cl) & $\boldsymbol{P}$ \\
\hline Radiotherapy & Yes vs No & $1.079(0.432-2.691)$ & 0.871 & $0.843(0.342-2.078)$ & 0.710 \\
\hline Mono-immunotherapy & Yes vs No & $1.786(0.758-4.212)$ & 0.185 & I & 1 \\
\hline Leading STM change & Decrease $<20 \%$ vs Decrease $\geq 20 \%$ & $2.892(0.976-8.568)$ & 0.055 & $0.510(0.193-1.350)$ & 0.175 \\
\hline Pretreatment NLR & $\geq 5$ vs $<5$ & I & l & $0.805(0.308-2.099)$ & 0.657 \\
\hline Posttreatment NLR & $\geq 5$ vs $<5$ & $0.144(0.048-0.427)$ & 0.001 & $0.122(0.042-0.352)$ & 0.001 \\
\hline \multirow{2}{*}{\multicolumn{2}{|c|}{ B. The Combination Score Multivariate Cox Analysis }} & \multicolumn{2}{|l|}{ PFS } & \multicolumn{2}{|l|}{ os } \\
\hline & & HR $(95 \% \mathrm{Cl})$ & $\mathbf{P}$ & HR (95\% Cl) & $\mathbf{P}$ \\
\hline Radiotherapy & Yes vs No & $1.049(0.4 \mid 7-2.643)$ & 0.919 & $0.807(0.329-1.976)$ & 0.630 \\
\hline Mono-immunotherapy & Yes vs No & $1.860(0.772-4.480)$ & 0.167 & l & 1 \\
\hline Combination Score & $\begin{array}{l}0 \\
1 \\
2\end{array}$ & $\begin{array}{l}3.661(1.136-11.792) \\
20.066(5.646-71.311)\end{array}$ & $\begin{array}{l}0.030 \\
<0.0001\end{array}$ & $\begin{array}{l}3.183(1.02-9.931) \\
22.299(6.38 I-77.924)\end{array}$ & $\begin{array}{l}0.046 \\
<0.0001\end{array}$ \\
\hline
\end{tabular}

Notes: Cox regression models were applied to find independent indicators associated with PFS and OS and estimate the hazard ratio (HR). Factors which were statistically significant in the univariate analysis were incorporated into the multivariate analysis. Figures are given as $\mathrm{HR}(95 \% \mathrm{Cl})$. A $P$ value of $<0.05$ was regarded statistically significant.

Abbreviations: PFS, progression-free survival; OS, overall survival; HR: hazard ratio; Cl, confidence interval.

elucidated. Traditionally, most factors that predict a response to ICIs are related to the characteristics of cancer cells and the tumour-associated microenvironment. PD-L1 expression, as the only approved predictive biomarker for PD-1 blockade in NSCLC, is currently widely applied. However, some patients do not respond well to ICIs despite positive PD-L1 expression. Nonetheless, patients with negative PD-L1 expression may still benefit from inhibitors. This could be because PD-L1 expression may vary over time and may be distinct in different tumour lesions. ${ }^{24} \mathrm{~A}$ second biopsy for PD-L1 expression reanalysis may not be feasible in every patient in a clinical setting to aid in patient selection. Another biomarker, TMB, has been found to be related to durable responses in multiple cancers treated with ICIs, including NSCLC. ${ }^{25-28}$ Similarly, TMB has several limitations, including the lack of standardization among the testing platforms used and involved genes. ${ }^{29}$ Although blood TMB as a circulating biomarker has shown a positive association with efficacy of immune checkpoint blockade, ${ }^{30}$ a fixed TMB threshold that defines a tumour with high TMB has not been confirmed. The high overall costs and expertise for the interpretation of genomic data also limit TMB assessment in routine companion diagnostics. Other biomarkers including representatives of the tumour microenvironment, such as TILs and the Teff score, which represents effector T-cell gene expression, are reported as positive predictors for ICI therapy. However, these biomarkers are expensive and to some extent lack a consensus regarding the detection and analysis methods and are not yet widely applied in clinical practice. Sometimes, easy combinations of these biomarkers may exclude some patients who might truly have durable responses and derive benefits from immunotherapy in terms of clinical outcomes. In the CheckMate 026 study, patients with both high TMB and high PD-L1 expression had a higher response rate than those with only one of these factors or none of the factors. However, in this trial, these patients accounted for only $10 \%$ of all NSCLC patients. ${ }^{31}$ In this study, the dynamics of early STMs and the NLR were identified as inexpensive, easily obtainable and repeatable as biomarkers, which is vital to optimize the clinical use of ICIs, identify the subset of patients most likely to derive a clinical benefit, and to help understand the potential molecular determinants of the immune response.

In general, as surrogate indicators of tumour burden, circulating biomarker analyses are cheap, readily obtainable and repeatable. Although STMs are currently not recommended for the diagnosis or management of 


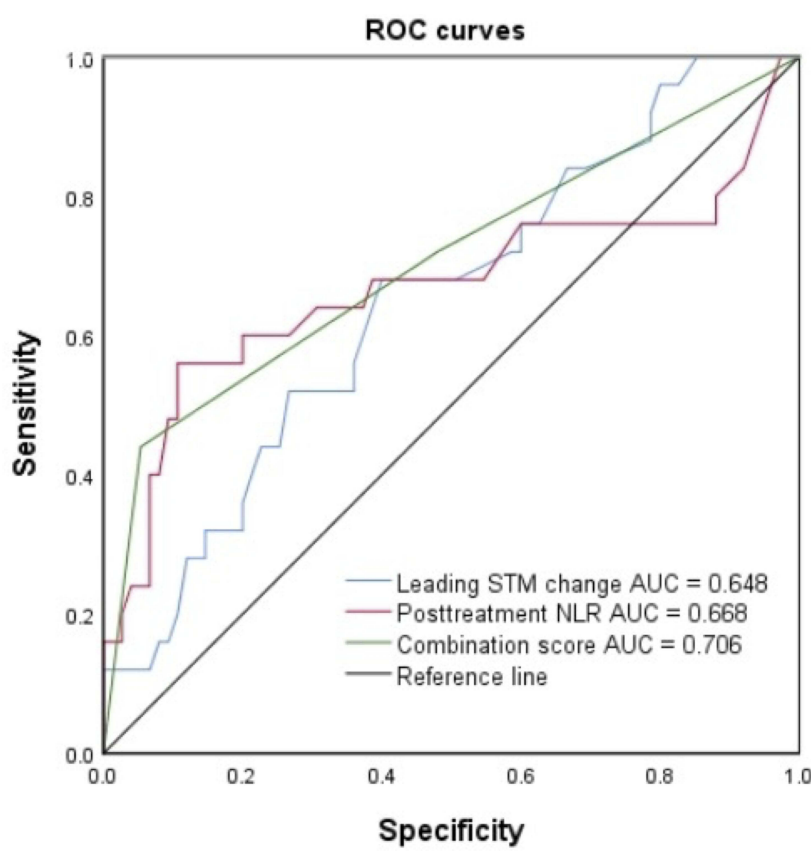

Figure 5 ROC curves for sensitivity and specificity according to leading STM change, posttreatment NLR and the combination score.

Abbreviations: STM, serum tumour marker; NLR, neutrophil-to-lymphocyte ratio; ROC, receiver operating curve; $A \cup C$, area under the curve.

advanced NSCLC, ${ }^{32}$ several biomarkers, including CEA, CYFRA21-1 and CA19-9, were shown to be predictive of PFS and OS. ${ }^{15-17}$ The pretreatment serum CEA or CYFRA 21-1 level was a predictive biomarker of PFS in patients with advanced NSCLC treated with nivolumab. ${ }^{15,16}$ Furthermore, a reduction in CEA or CYFRA21-1 levels greater than $20 \%{ }^{33}$ or a meaningful reduction in $>2$ of 4 biomarkers (CEA, CA125, CYFRA21-1, and SCC-Ag), ${ }^{34}$ as a dynamic index, may serve as a reliable early marker of high ORR and prolonged PFS and OS. More recently, a decreased leading STM was similarly associated with longer PFS in NSCLC patients treated with monoimmunotherapy, ${ }^{17}$ as well as in those treated with chemoimmunotherapy and monoimmunotherapy maintenance. ${ }^{35}$ In the present study, we used a panel of 5 STMs, including CEA, CYFRA 21-1, NSE, CA19-9, and CA125, that were linked to poor outcomes of NSCLC patients to define the leading STM.

In addition, host biomarkers could help select patients that might derive a benefit from ICI therapy. ECOG performance status, inflammatory factors, routine laboratory parameters, such as lactate dehydrogenase and peripheral blood cell counts, and gut microbiota, ${ }^{9-11}$ have been found to be associated with a durable response to immunotherapy. Considering that these markers represent the status of host immunity and inflammation, combined analysis of host biomarkers, tumour genomic biomarkers, and tumour microenvironment biomarkers can comprehensively and precisely predict sensitivity to ICI therapy.

Several studies have reported that a high pretreatment NLR is a prognostic marker that strongly correlates with poor survival in NSCLC patients treated with nivolumab. ${ }^{36,37}$ However, in our study, the pretreatment NLR was not a significant predictor of efficacy or outcome. Suh KJ et al reported that an NLR $>5$ after 6 weeks of treatment was associated with poor PFS and OS in antiPD-1-treated advanced NSCLC, ${ }^{38}$ which was consistent with our results that a posttreatment NLR $<5$ was a stronger predictor of excellent response and favourable outcome. This indicates that a change in the posttreatment NLR, but not in the initial NLR, can be exploited as an accurate biomarker to predict patient response to ICI treatment. Recently, the ratio between pretreatment and posttreatment NLR was shown to be significantly correlated with PFS (6.2 months for NLR $\geq 1$ vs 3.0 months for NLR $<10){ }^{38}$ Thus, we used the posttreatment NLR as a dynamic marker of the NLR for further analysis. As expected, the combination score incorporating the dynamics of the early leading STM and the NLR can aid in selecting patients who are responsive to immunotherapy and who would exhibit a durable benefit. To the best of our knowledge, this is the first study that combines changes in an STM and the NLR as predictive biomarkers in patients with advanced NSCLC treated with PD-1/PD-L1 inhibitors.

We further found that the predictive potency of these combined serum markers showed consistent results in a subset of patients with no response at the first radiological assessment. Although most patients who exhibited long-term survival were responders, a small group of patients with SD or progressive disease also derived a long-term benefit from immunotherapy. Recent studies have indicated that patients with pseudoprogression or dissociated response exhibited better survival than those with true progression. ${ }^{32,39-42}$ Considering the possibility of these atypical response patterns, classical imaging response criteria, such as RECIST1.1, have been shown to frequently underestimate or overestimate the therapeutic effect of ICIs. ${ }^{43}$ Similar to the previously reported predictor, leading STM, ${ }^{17}$ the combination score is designed for all patients, including responders and nonresponders, complements radiological assessment, and helps to identify nonresponders who might experience long-term benefits. 
Furthermore, the AUC demonstrated that the combination score has greater predictive power than changes in the posttreatment NLR or leading STM alone.

Our study still has several limitations. First, this study was limited by its retrospective nature and its rather small, predominantly Chinese population. Second, we only used interior validation and subgroup analyses, and thus, further external validation is needed. Third, the results may have been influenced by the method used for selecting the cutoff points. Here, a leading STM decrease $\geq 20 \%$ and an NLR $<5$ were selected as thresholds to identify changes in the leading STM and NLR, respectively, according to previous reports. Fourth, only patients who received more than 2 cycles of ICI treatment were enrolled in this study, which may have increased selection bias. Fifth, the STM and NLR were measured retrospectively after a patient had already been treated with ICI therapy, while traditional biomarkers, such as PD-L1 expression or TMB, allow an a priori prognostic analysis. A general limitation of STM evaluation is that none of the included markers is specific to a certain cancer entity. Finally, other factors, such as various chronic or acute conditions (infection, renal function impairment or trauma), apart from malignancies and concomitant steroid administration, may influence STM concentrations and NLR values. ${ }^{44-47}$ Future research should focus on baseline combinations of markers of total metabolic tumour volume, molecular and expression profiling of the tumour, characteristics of the tumour microenvironment, and the immunological status of the host. Additional dynamic combination markers measured throughout the early stage of immunotherapy can supplement radiological restaging evaluation.

\section{Conclusion}

In conclusion, an easy, affordable, and novel combination score based on routinely available dynamics of a leading STM and the NLR was first developed and validated and is a practical clinical tool for predicting the response to immune checkpoint blockade in advanced NSCLC. Large-size, prospective, multicentre clinical trials are warranted as the next step.

\section{Ethics Approval and Informed Consent}

The study protocol was conducted in accordance with the Declaration of Helsinki and was approved by the independent research ethics committee of The First Affiliated
Hospital of Shandong First Medical University (NO: YXLL-KY-2020-007). The study was conducted in an entirely retrospective manner without an experimental approach or additional patient contact. Only patient data assessed in routine clinical practice were analysed. Patient data were collected in an anonymized fashion and were safely electronically stored such that only the authors had access to the data. No identifiable patient data have been or will ever be published by the authors. Thus, according to ethics committee approval, no patient consent was required for participation in this study.

\section{Acknowledgments}

We sincerely thank the patients for their contribution to the publication of this study. The abstract of this paper was presented at the 2021 ASCO Annual Meeting as a poster presentation with interim findings. The poster's abstract was published in "Poster Abstracts" in the Journal of Clinical Oncology: https://ascopubs.org/doi/abs/10.1200/ JCO.2021.39.15_suppl.e21095

\section{Author Contributions}

Yin Tang, Ya-hong Sun, and Jun Wang were involved in the design of the original study and drafted the manuscript. Yin Tang, Yu Cui, Lin-lin Li, Ya-ping Guan, and Rui Jiang were involved in data curation, data analysis and editing of the manuscript. Dong-feng Feng, Bei-bei Yin, Xue-feng Liang, Jing Yin and Jing Liang reviewed and edited the manuscript. All authors contributed to data analysis, drafting or revising the article, have agreed on the journal to which the article will be submitted, gave final approval of the version to be published, and agree to be accountable for all aspects of the work.

\section{Funding}

This study was supported by the National Natural Science Foundation of China (Grant No. 81572875), the CSCOMSD Cancer Research Foundation (Grant No. Y-MSD2020-0350), CSCO-PILOT Cancer Research Foundation (Grant No. Y-2019AZMS-0440), the Wu Jieping Medical Foundation for Clinical Scientific Research (Grant No. 320.6750.2020-12-16), the Major Science and Technology Innovation Project of Shandong Province (2018CXGC1220), the Natural Science Foundation of Shandong Province (Grant No. ZR202102190539), and the Innovation Project of Shandong Academy of Medical Sciences. 


\section{Disclosure}

The authors report no conflicts of interest in this work.

\section{References}

1. Chen W, Zheng R, Baade P, et al. Cancer statistics in China, 2015. CA Cancer J Clin. 2016;66(2):115-132. doi:10.3322/caac.21338

2. Torre LA, Siegel RL, Jemal A. Lung Cancer Statistics. Adv Exp Med Biol. 2016;893:1-19.

3. Siegel RL, Miller KD, Jemal A. Cancer statistics, 2020. CA Cancer J Clin. 2020;70(1):7-30. doi:10.3322/caac.21590

4. Bodor JN, Boumber Y, Borghaei H. Biomarkers for immune checkpoint inhibition in non-small cell lung cancer (NSCLC). Cancer. 2020;126(2):260-270. doi:10.1002/cncr.32468

5. Hellmann MD, Ciuleanu TE, Pluzanski A, et al. Nivolumab plus ipilimumab in lung cancer with a high tumor mutational burden. N Engl J Med. 2018;378:2093-2104. doi:10.1056/NEJMoa1801946

6. Brambilla E, Le Teuff G, Marguet S, et al. Prognostic effect of tumor lymphocytic infiltration in resectable non-small-cell lung cancer. J Clin Oncol. 2016;34:1223-1230. doi:10.1200/JCO.2015.63.0970

7. Skoulidis F, Goldberg ME, Greenawalt DM, et al. STK11/LKB1 mutations and PD-1 inhibitor resistance in KRAS-mutant lung adenocarcinoma. Cancer Discov. 2018;8:822-835. doi:10.1158/ 2159-8290.CD-18-0099

8. Fehrenbacher L, Spira A, Ballinger M, et al. Atezolizumab versus docetaxel for patients with previously treated non-small-cell lung cancer (POPLAR): a multicentre, open-label, Phase 2 randomised controlled trial. Lancet. 2016;387:1837-1846.

9. Dall'Olio FG, Maggio I, Massucci M, Mollica V, Fragomeno B, Ardizzoni A. ECOG performance status $\geq 2$ as a prognostic factor in patients with advanced non small cell lung cancer treated with immune checkpoint inhibitors-A systematic review and metaanalysis of real world data. Lung Cancer. 2020;145:95-104. doi:10.1016/j.lungcan.2020.04.027

10. Peng L, Wang Y, Liu F, et al. Peripheral blood markers predictive of outcome and immune-related adverse events in advanced non-small cell lung cancer treated with PD-1 inhibitors. Cancer Immunol Immunother. 2020;69(9):1813-1822. doi:10.1007/s00262-020-02585-w

11. Jin Y, Dong H, Xia L, et al. The Diversity of Gut Microbiome is Associated With Favorable Responses to Anti-Programmed Death 1 Immunotherapy in Chinese Patients With NSCLC. $J$ Thorac Oncol. 2019;14(8):1378-1389. doi:10.1016/j.jtho.2019.04.007

12. Ladbury CJ, Rusthoven CG, Camidge DR, Kavanagh BD, Nath SK. Impact of radiation dose to the host immune system on tumor control and survival for stage III non-small cell lung cancer treated with definitive radiation therapy. Int J Radiat Oncol Biol Phys. 2019;105 (2):346-355. doi:10.1016/j.ijrobp.2019.05.064

13. Mendes F, Antunes C, Abrantes AM, et al. Lung cancer: the immune system and radiation. $B r \quad J$ Biomed Sci. 2015;72(2):78-84. doi:10.1080/09674845.2015.11666801

14. Templeton AJ, McNamara MG, Seruga B, et al. Prognostic role of neutrophil-to-lymphocyte ratio in solid tumors: a systematic review and meta-analysis. $J$ Natl Cancer Inst. 2014;106(6):dju124. doi:10.1093/jnci/dju124

15. Kataoka Y, Hirano K, Narabayashi T, et al. Carcinoembryonic antigen as a predictive biomarker of response to nivolumab in non-small cell lung cancer. Anticancer Res. 2018;38(1):559-563.

16. Shirasu H, Ono A, Omae K, et al. CYFRA 21-1 predicts the efficacy of nivolumab in patients with advanced lung adenocarcinoma. Tumour Biol. 2018;40(2):1010428318760420. doi:10.1177/1010428318760420

17. Lang D, Horner A, Brehm E, et al. Early serum tumor marker dynamics predict progression-free and overall survival in single PD-1/PD-L1 inhibitor treated advanced NSCLC-A retrospective cohort study. Lung Cancer. 2019;134:59-65. doi:10.1016/j. lungcan.2019.05.033
18. Zhang QY, Wang FX, Jia KK, Kong LD. Natural Product Interventions for Chemotherapy and Radiotherapy-Induced Side Effects. Front Pharmacol. 2018;9:1253. doi:10.3389/fphar.2018.01253

19. He Y, Rozeboom L, Rivard CJ, et al. PD-1, PD-L1 Protein Expression in Non-Small Cell Lung Cancer and Their Relationship with Tumor-Infiltrating Lymphocytes. Med Sci Monit. 2017;23:1208-1216. doi:10.12659/MSM.899909

20. Borghaei H, Paz-Ares L, Horn L, et al. Nivolumab versus docetaxel in advanced nonsquamous non-small-cell lung cancer. $N$ Engl J Med. 2015;373(17):1627-1639. doi:10.1056/NEJMoa1507643

21. Brahmer J, Reckamp KL, Baas P, et al. Nivolumab versus docetaxel in advanced squamous-cell non-small-cell lung cancer. $N$ Engl J Med. 2015;373(2):123-135. doi:10.1056/NEJMoa1504627

22. Herbst RS, Baas P, Kim DW, et al. Pembrolizumab versus docetaxel for previously treated, PD-L1-positive, advanced non-small-cell lung cancer (KEYNOTE-010): a randomised controlled trial. Lancet. 2016;387(10027):1540-1550. doi:10.1016/S0140-6736(15) 01281-7

23. Vokes EE, Ready N, Felip E, et al. Nivolumab versus docetaxel in previously treated advanced non-small-cell lung cancer (CheckMate 017 and CheckMate 057): 3-year update and outcomes in patients with liver metastases. Ann Oncol. 2018;29(4):959-965. doi:10.1093/ annonc/mdy041

24. Topalian SL, Hodi FS, Brahmer JR, et al. Safety, activity, and immune correlates of anti-PD-1 antibody in cancer. $N$ Engl $J$ Med. 2012;366(26):2443-2454. doi:10.1056/NEJMoa1200690

25. Snyder A, Makarov V, Merghoub T, et al. Genetic basis for clinical response to CTLA-4 blockade in melanoma. $N$ Engl J Med. 2014;371 (23):2189-2199. doi:10.1056/NEJMoa1406498

26. Rizvi NA, Hellmann MD, Snyder A, et al. Cancer immunology. Mutational landscape determines sensitivity to PD-1 blockade in non-small cell lung cancer. Science. 2015;348(6230):124-128. doi:10.1126/science.aaa1348

27. Le DT, Durham JN, Smith KN, et al. Mismatch repair deficiency predicts response of solid tumors to PD-1 blockade. Science. 2017;357(6349):409-413. doi:10.1126/science.aan6733

28. Yarchoan M, Hopkins A, Jaffee EM. Tumor Mutational Burden and Response Rate to PD-1 Inhibition. $N$ Engl J Med. 2017;377 (25):2500-2501. doi:10.1056/NEJMc1713444

29. Herbst RS, Soria JC, Kowanetz M, et al. Predictive correlates of response to the anti-PD-L1 antibody MPDL3280A in cancer patients. Nature. 2014;515(7528):563-567. doi:10.1038/nature14011

30. Hellmann MD, Nathanson T, Rizvi H, et al. Genomic Features of Response to Combination Immunotherapy in Patients with Advanced Non-Small-Cell Lung Cancer. Cancer Cell. 2018;33(5):843-852.e4. doi:10.1016/j.ccell.2018.03.018

31. Carbone DP, Reck M, Paz-Ares L, et al. First-Line Nivolumab in Stage IV or Recurrent Non-Small-Cell Lung Cancer. $N$ Engl J Med. 2017;376(25):2415-2426. doi:10.1056/NEJMoa1613493

32. Planchard D, Popat S, Kerr K, et al. Metastatic non-small cell lung cancer: ESMO Clinical Practice Guidelines for diagnosis, treatment and follow-up. Ann Oncol. 2018;29(Suppl4):iv192-iv237.

33. Dal Bello MG, Filiberti RA, Alama A, et al. The role of CEA, CYFRA21-1 and NSE in monitoring tumor response to Nivolumab in advanced non-small cell lung cancer (NSCLC) patients. $J$ Transl Med. 2019;17(1):74. doi:10.1186/s12967-019-1828-0

34. Zhang Z, Yuan F, Chen R, et al. Dynamics of serum tumor markers can serve as a prognostic biomarker for Chinese advanced non-small cell lung cancer patients treated with immune checkpoint inhibitors. Front Immunol. 2020;11:1173. doi:10.3389/ fimmu.2020.01173

35. Lang D, Haslinger W, Akbari K, et al. Serum Tumor Marker Dynamics as Predictive Biomarkers in NSCLC Chemo-Immunotherapy and Mono-Immunotherapy Maintenance: a Registry-Based Descriptive Study. Lung Cancer. 2020;11:113-121. 
36. Diem S, Schmid S, Krapf M, et al. Neutrophil-to-Lymphocyte ratio (NLR) and Platelet-to-Lymphocyte ratio (PLR) as prognostic markers in patients with non-small cell lung cancer (NSCLC) treated with nivolumab. Lung Cancer. 2017;111:176-181. doi:10.1016/j.lungcan.2017.07.024

37. Bagley SJ, Kothari S, Aggarwal C, et al. Pretreatment neutrophil-tolymphocyte ratio as a marker of outcomes in nivolumab-treated patients with advanced non-small-cell lung cancer. Lung Cancer. 2017;106:1-7. doi:10.1016/j.lungcan.2017.01.013

38. Suh KJ, Kim SH, Kim YJ, et al. Post-treatment neutrophil-to-lymphocyte ratio at week 6 is prognostic in patients with advanced non-small cell lung cancers treated with anti-PD-1 antibody. Cancer Immunol Immunother. 2018;67(3):459-470. doi:10.1007/s00262-017-2092-x

39. Rizvi NA, Mazières J, Planchard D, et al. Activity and safety of nivolumab, an anti-PD-1 immune checkpoint inhibitor, for patients with advanced, refractory squamous non-small-cell lung cancer (CheckMate 063): a phase 2, single-arm trial. Lancet Oncol. 2015;16(3):257-265. doi:10.1016/S1470-2045(15)70054-9

40. Kazandjian D, Keegan P, Suzman DL, Pazdur R, Blumenthal GM. Characterization of outcomes in patients with metastatic non-small cell lung cancer treated with programmed cell death protein 1 inhibitors past RECIST version 1.1-defined disease progression in clinical trials. Semin Oncol. 2017;44(1):3-7. doi:10.1053/j.seminoncol.2017.01.001

41. Gandara DR, von Pawel J, Mazieres J, et al. Atezolizumab Treatment Beyond Progression in Advanced NSCLC: results From the Randomized, Phase III OAK Study. J Thorac Oncol. 2018;13 (12):1906-1918. doi:10.1016/j.jtho.2018.08.2027
42. Tazdait M, Mezquita L, Lahmar J, et al. Patterns of responses in metastatic NSCLC during PD-1 or PDL-1 inhibitor therapy: comparison of RECIST 1.1, irRECIST and iRECIST criteria. Eur J Cancer. 2018;88:38-47. doi:10.1016/j.ejca.2017.10.017

43. Dimitrakopoulou-Strauss A. Monitoring of patients with metastatic melanoma treated with immune checkpoint inhibitors using PET-CT. Cancer Immunol Immunother. 2019;68(5):813-822. doi:10.1007/ s00262-018-2229-6

44. Chen J, Tao F, Zhang B, et al. Elevated Squamous Cell Carcinoma Antigen, Cytokeratin 19 Fragment, and Carcinoembryonic Antigen Levels in Diabetic Nephropathy. Int $J$ Endocrinol. 2017;2017:5304391. doi:10.1155/2017/5304391

45. Anderson BJ, Reilly JP, Shashaty MGS, et al. Admission plasma levels of the neuronal injury marker neuron-specific enolase are associated with mortality and delirium in sepsis. J Crit Care. 2016;36:18-23. doi:10.1016/j.jcrc.2016.06.012

46. Kwon YJ, Lee HS, Shim JY, Lee YJ. Serum carcinoembryonic antigen is positively associated with leukocyte count in Korean adults. J Clin Lab Anal. 2018;32(3):e22291. doi:10.1002/jcla.22291

47. Weber TH, Kerttula Y. Carcinoembryonic antigen (CEA) in blood in cases of pneumonia. Scand J Infect Dis. 1986;18(6):547-550. doi:10.3109/00365548609021660

\section{Publish your work in this journal}

Cancer Management and Research is an international, peer-reviewed open access journal focusing on cancer research and the optimal use of preventative and integrated treatment interventions to achieve improved outcomes, enhanced survival and quality of life for the cancer patient.
The manuscript management system is completely online and includes a very quick and fair peer-review system, which is all easy to use. Visit http://www.dovepress.com/testimonials.php to read real quotes from published authors. 